\title{
Review
}

\section{Diabetes and chronic infections}

\author{
Kapur $\mathbf{A}^{1}$ \\ Journal of the Ceylon College of Physicians, 2015, 46, 34-38
}

\section{Diabetes and infections}

The improvement in human health that has been achieved in the last two decades ${ }^{1}$ has been paralleled by a rise in chronic non-communicable diseases (NCDs). Over one-third of adults worldwide are overweight ${ }^{2}$, about $40 \%$ hypertensive ${ }^{3-4}$, and approximately 700 million people dysglycaemic (diabetes mellitus [DM] and impaired glucose tolerance [IGT]). ${ }^{5}$ Eighty percent of people with DM live in middle-income countries, which are host to $88 \%$ of deaths due to DM. ${ }^{5}$

In addition to its association with hypertension, obesity, and lipid disorders, diabetes also acts as a fertile ground for several acute and chronic infections. Infections in people with DM are associated with increased risk, severity and poor outcomes such as hospitalisation and deaths. Through release of proinflammatory immune mediators, infections worsen insulin resistance and cause metabolic decompensation, setting a vicious downward spiral. Through immunological as well as non-immunological mechanisms, chronic infections may also precipitate the onset of DM in those at risk. Respiratory tract, genitourinary tract, skin and soft tissues are particularly vulnerable. The interaction between DM and infectious diseases is particularly relevant in the developing world, with the so-called 'double burden.'

This review visits the interactions between DM and chronic infections and focuses on some common infections.

\section{Increased risk of infections}

It is generally accepted that people with DM are at increased risk of all types of infections, attributed to compromised host defences, due to the long-term effects of hyperglycaemia on physical barriers and both humoral and cell-mediated immune responses. There are, however, few studies that have actually investigated the risk of infection in people with DM, since they are difficult to conduct and interpret.

In a large, population-based, retrospective study in Ontario, Canada, Shah and Hux ${ }^{6}$ found that the risk

\footnotetext{
${ }^{1}$ Chairman, World Diabetes Foundation, Denmark.

E-mail: dranilkapur@gmail.com
}

ratio for an infectious disease hospitalization or physician claim in the DM cohort was 1.21 versus the control cohort. There was a significantly higher rate of death attributable to infectious diseases among people with DM, and a significantly higher death rate within 5 days of a physician claim for infectious disease. A prospective study in the Netherlands found an increased susceptibility to lower respiratory tract, genito-urinary tract and skin and mucous membrane infections in DM compared to hypertension. ${ }^{7}$ A UK study found considerable morbidity and mortality due to infections in the elderly with DM. ${ }^{8}$

In addition to this broad susceptibility to infection, there is also evidence for susceptibility to specific infections in DM. These include carbuncles, emphysematous pyelonephritis, invasive otitis externa, emphysematous cholecystitis, pyelonephritis, Fournier's gangrene and rhinocerebral mucormycosis, ${ }^{9}$ which are all rare in non-diabetics. DM also increases the likelihood of infections caused by certain bacteria such as Staphylococcus aureus, Burkholderia mallei (melioidosis), and Mycobacterium tuberculosis.

\section{Pathogenesis and immunology}

The mechanism behind the increased susceptibility is not fully understood. Microvascular damage is mentioned, but there is little evidence to support this hypothesis. Non-enzymatic glycosylation of tissue proteins may alter their functions (e.g., bronchociliary functions and reduce bronchial reactivity). In long standing DM, autonomic neuropathy may induce changes in the cough threshold, thereby increasing stagnation and reducing clearance of secretions and bacterial load, leading to infection. Similarly, DMinduced cystopathy, characterized by impaired sensation of bladder fullness, leads to overstretched bladder, reduced bladder contractility, increased residual urine and impaired urinary flow leading to urine stagnation. Contractile dysfunctions of biliary and gastrointestinal tract may promote infections at the respective sites. Hyperglycaemia, by making glucose available in the respiratory tree, subcutaneous tissues and genitourinary tract, supports growth of pathogens. ${ }^{10,11}$

\section{Respiratory tract infections}

People with DM seem to be particularly prone to 
respiratory infections. Respiratory infections resulting in pneumonia seem rather common in people with DM. A case-control study of community-acquired pneumococcal pneumonia found a $50 \%$ higher risk in people with DM. ${ }^{13}$ Amongst patients hospitalised with influenza pneumonia, the odds ratio (OR) for having DM was 4.72. ${ }^{14}$ Pneumonia caused by opportunistic pathogens such as Klebsiella, Pseudomonas, Burkholderia mallei and many fungi are also more common in people with DM. ${ }^{12,15}$ Other pneumonia-causing pathogens more commonly seen in association with DM are Mycoplasma pneumoniae, Chlamydia pneumoniae, Legionella spp. and Hemophilus influenzae.

During the outbreak of Severe Acute Respiratory Syndrome (SARS), a known history of DM and ambient hyperglycaemia were independent predictors for death and morbidity. ${ }^{16,17}$ In the recent outbreak of Middle East Respiratory Syndrome (MERS), over two-thirds (68\%) of all case fatalities occurred in patients with co-morbid DM. ${ }^{18}$

\section{Diabetes and tuberculosis (TB)}

DM and TB have co-existed for thousands of years. As noted by Root ${ }^{19}$ "in the latter half of the $19^{\text {th }}$ century the diabetic patient appeared doomed to die of pulmonary TB (PTB) if he succeeded in escaping coma". The availability of effective treatment for TB and DM and successful public health measures in the developed world to combat TB have made the association irrelevant: TB did not exist where DM was present and DM was uncommon where TB was endemic. In the last one-and-half decade, the dramatic rise of DM in low- and middle-income countries has brought this association to the forefront again. A systematic review ${ }^{20}$ found that DM is associated with an increased risk of TB; the pooled relative risk (RR) of TB associated with DM was 3.1 and in case-control studies the OR of TB ranged from 1.16 to 7.83. The risk was higher in countries with a high background incidence of TB. Stevenson et $\mathrm{al}^{21}$ reported similar findings, with RR estimates ranging from 1.5 to 7.8 and a modelling study ${ }^{22}$ based on data for 2000 from India reported that DM accounted for nearly $15 \%$ of PTB and $20 \%$ of smear-positive PTB.

A more recent paper reviewed studies that had screened people with DM for active TB and studies that had screened TB patients for DM and assessed the yield of screening. Screening for TB in persons with DM showed high rates of TB prevalence, increasing with rising TB prevalence in the underlying population as well as with DM severity. Screening patients with TB for DM also yielded high prevalence rates of DM. ${ }^{23,24}$

A study from Mexico reported that TB patients with DM co-morbidity have more severe clinical manifestations and poor outcomes as determined by haemoptysis (aOR1.66); higher probability of delayed sputum clearance (aOR 1.43); treatment failure (aOR 2.93); recurrence (aOR 1.76); and relapse (aOR 1.92). ${ }^{25}$

\section{Skin and soft tissue infections}

Skin and soft tissue infections (SSTIs) cause considerable morbidity and mortality in persons with DM. ${ }^{26,29}$ While complications of DM, such as sensory neuropathy and vascular insufficiency, compound the picture, it is primarily the metabolic disturbances and the resultant inadequate immune response that predispose people with DM to chronic infected wounds and severe tissue destruction. ${ }^{30,31}$ It is well known that neuropathic foot ulcers in patients with leprosy generally do not develop fulminant infections as commonly seen with neuropathic diabetic foot ulcers - suggesting that the diabetic milieu enhances the risk of infections. Patients with life-threatening infection have a history of foot infection for more than a month. Culture reports vary but over two third of the cultures reveal polymicrobial infection. In one recent report from India, Gram negative infections were more common and the most common pathogens isolated were Pseudomonas aeruginosa (20.1\%), Staphylococcus aureus (17.2\%) and Escherichia coli (16.3\%). ${ }^{32}$ Antimicrobial-resistant pathogens, especially methicillin-resistant S. Aureus (MRSA), are increasingly isolated in nosocomial infections. MRSA causes $20 \%$ to $50 \%$ of DMassociated foot infections and results in worse outcomes than other pathogens. ${ }^{33-35}$ SSTIs occur at all anatomic sites, but the foot is most frequently affected in diabetic patients. Severe hand infections in people with DM can be equally disastrous and result in the tropical hand syndrome. ${ }^{36}$

\section{Genitourinary Infections}

DM doubles the risk of UTI, ${ }^{37,38}$ and is related to duration of DM and presence of glycosuria. The risk for upper urinary tract and chronic infections is higher. A rare unusual presentation of UTI in people with DM is emphysematous pyelonephritis. Women are at greater risk of UTI than men and frequency of sexual activity is a risk factor. People with DM with bladder dysfunction resulting in urinary retention or obstruction to urinary flow such as urinary calculi or prostate enlargement are at higher risk of UTI. Asymptomatic bacteriuria is more common in people with DM, but does not necessarily increase the risk of symptomatic infection, except during pregnancy, prior to uro-genital or gastrointestinal surgery, or in those with marked urinary retention and does not otherwise merit treatment. ${ }^{38}$ The approval of new anti-diabetic drugs called sodium glucose co-transporter 2 (SGLT2)inhibitors, which work through prevention of glucose reuptake in the renal tubules resulting in glycosuria, 
are associated with increased frequency of UTI amongst people receiving them, despite improved metabolic control. ${ }^{39,40}$ Apart from the higher risk, in general, the epidemiology and clinical manifestations of UTIs among men and women with DM is similar to those without $D M,{ }^{38,41}$ except for less frequent flank pain. ${ }^{42}$ The bacteriology and antibiotic susceptibility patterns also do not, in general, differ from those without DM. ${ }^{43}$

Women with pre-gestational or gestational DM may be at even higher risk of UTI compared to both pregnant women without DM and non-pregnant women with $\mathrm{DM}$, and may require special attention due to higher risk of pregnancy complication as a consequence of UTI. ${ }^{44}$ Unfortunately the subject has not been researched. Similarly there is very little high quality information available on the risk of postpartum genital infections in women with DM. Given the rising burden of DM during pregnancy particularly in the developing world ${ }^{5}$ one would expect greater interest on the subject.

People with DM, especially those with poor control are prone to develop genital mycotic infections: vulvovaginal candidiasis in women and candida balanitis almost exclusively in uncircumcised men. Candida albicans is the most common pathogen causing balanitis and is also the dominant cause of vulvovaginal candidiasis in women with DM, although C. glabrata is also a prominent pathogen in women with type 2 DM mellitus. High glucose promotes yeast attachment and growth, and also interferes with the host immune responses. In patients with hyperglycemia, risk is increased for not only incident infection but also for recurrence, underscoring the key role of establishing and maintaining euglycemia in the management of genital mycotic infections in people with DM. As with UTI, the use of SGLT2 inhibitors is accompanied by a significantly ( 4 to 6 fold) increased risk of vulvovaginitis or balanitis in people with DM.

\section{Chronic periodontitis}

Periodontitis, an oral infection with primarily anaerobic gram-negative bacteria, causes chronic gingival inflammation, bone destruction, loss of attachment and eventually the loss of teeth in severe cases. Microbial flora of the dental plaques produce endotoxins in the form of lipopolysaccharides (LPS) which are responsible for host-mediated tissue destructive immune response. Periodontitis is no longer regarded as a mere oral disease but a condition with significant systemic effects including worsening of DM, cardiovascular and renal disease.

Oral diseases affected 3.9 billion people globally in 2010. Untreated caries was the most prevalent condition evaluated for the entire Global Burden of
Disease Study (GBD 2010) with a global prevalence of $35 \%$ for all ages combined. Severe periodontitis and untreated caries in deciduous teeth were the 6th and 10th most prevalent conditions, affecting $11 \%$ and $9 \%$ of the global population. ${ }^{45}$ Epidemiological data confirm that DM is a major risk factor for periodontitis. ${ }^{46}$ Evidence also supports an increased risk for the development of type 2 (and possibly gestational) DM in patients with severe periodontitis. ${ }^{47}$ In DM patients, there is a direct and dose-dependent relationship between periodontitis severity and DM complications. Current evidence suggests that periodontal disease adversely affects DM outcomes; ${ }^{47}$ incidence of macroalbuminuria and end-stage renal disease is increased twofold and threefold respectively and the risk of cardiorenal mortality (ischemic heart disease and diabetic nephropathy combined) is three times higher in people with DM who also have severe periodontitis compared to those without severe periodontitis. ${ }^{48}$

The effect of periodontal therapy on changing DM outcomes has not been clearly established. However, a recent meta-analysis of randomized controlled clinical trials including periodontal therapy for diabetic subjects, a metabolic outcome, an untreated control group, and follow-up of at least 3 months showed a mean $0.36 \%$ lowering of $\mathrm{HbA} 1 \mathrm{c}(95 \% \mathrm{Cl}, 0.54-0.19)$ compared to no periodontal treatment $(p<0.0001) .{ }^{49}$ The modest but consistent reduction in HbA1c observed as a result of periodontal therapy in subjects with type 2 DM approximates that of adding a second line oral anti diabetic drug in patients not controlled with one drug. In addition periodontitis and DM also share common risk factors such as poor nutrition, consumption of unhealthy high sugar foods and smoking.

\section{Helicobacter pylori infection and DM}

Helicobacter pylori ( $H$. pylori) infection is rather common particularly in the developing countries where around $80 \%$ of the population maybe infected early in life through a feco-oral route. The potential link between $H$. pylori infection and type 2 DM was first reported in 1989. ${ }^{50,53}$ The mechanism behind the increased risk of DM caused by $H$. pylori infection is speculative and unclear. Most studies agree that eradicating $H$. pylori in patients with DM is difficult and re-infection rates are higher. Treatment of $H$. pylori infection does not seem to improve glucose control. Thus while the association seems interesting no conclusions can be drawn on the mechanism or the significance of the association till more evidence on the link becomes available.

\section{References}

1. Horton R. GBD 2010: understanding disease, injury, and risk. The Lancet 2012; 380: 2053-4. 
2. Obesity and overweight. Fact sheet $\mathrm{N}^{\circ} 311, \mathrm{WHO}$. http:// www.who.int/mediacentre/factsheets/fs311/en/

3. http://www.fao.org/news/story/en/item/161819/icode

4. Raised blood pressure. Global Health Observatory (GHO). http://www.who.int/gho/ncd/risk_factors/blood_pressure_ prevalence_text/en/

5. IDF Diabetes Atlas 6th Edition. 2013. IDF Diabetes Atlas: www.idf.org/diabetesatlas

6. Shah BR, Hux JE. Quantifying the risk of infectious diseases for people with diabetes. Diabetes Care 2003; 26: 510513. * (provides a good overview of the burden of common infections in people with diabetes).

7. Muller LM, Gorter KJ, Hak E, et al. Increased risk of common infections in patients with type 1 and type 2 diabetes mellitus. Clin Infect Dis 2005; 41: 281-8.

8. McDonald $\mathrm{HI}$, Nitsch D, Millett ER, et al. New estimates of the burden of acute community-acquired infections among older people with diabetes mellitus: a retrospective cohort study using linked electronic health records. Diabet Med. doi:10.1111/dme.12384 (2013).

9. Joshi N, Caputo GM, Weitekamp MR, Karchmer AW. Infections in patients with diabetes mellitus. N Engl J Med 1999; 341: 1906-12.

10. Philips BJ, Meguer JX, Redman J, Baker EH. Factors determining the appearance of glucose in upper and lower respiratory tract secretions. Intensive Care Med. 2003; 29: 2204-10.

11. Philips BJ, Redman J, Brennan A, Wood D, Holliman R, Baines $D$, Baker $E H$. Glucose in bronchial aspirates increases the risk of respiratory MRSA in intubated patients. Thorax 2005; 60: 761-4.

12. Peleg AY, Weerarathna T, McCarthy JS, Davis TM. Common infections in diabetes: pathogenesis, management and relationship to glycemic control. Diabetes Metabolism Research and Reviews 2007; 23: 3-13.

13. Thomsen RW, Hundborg $\mathrm{HH}$, Lervang $\mathrm{HH}$, Johnsen SP, Schonheyder HC, Sorensen HT. Diabetes mellitus as a risk and prognostic factor for community-acquired bacteremia due to enterobacteria: a 10-year, population-based study among adults. Clinical Infectious Diseases 2005; 40: 628-31.

14. Allard R, Leclerc P, Tremblay C, Tannenbaum TN. Diabetes and the severity of pandemic influenza A (H1N1) infection. Diabetes Care 2010; 33: 1491-3.

15. Santhosh YL, Ramanath KV, Naveen MR. Fungal Infections in diabetes mellitus: an Overview. International Journal of Pharmaceutical Sciences Review and Research 2011; 7: 221-5.

16. Yang JK, Feng Y, Yuan MY, et al. Plasma glucose levels and diabetes are independent predictors for mortality and morbidity in patients with SARS. Diabetic Medicine 2006; 23: 623-8

17. Booth CM, Matukas LM, Tomlinson GA, et al. Clinical Features and Short-term Outcomes of 144 Patients with SARS in the Greater Toronto Area. JAMA 2003; 289: 2801-9.

18. Assiri A, Al-Tawfiq JA, Al-Rabeeah A, et al. Epidemiological, demographic, and clinical characteristics of 47 cases of Middle East respiratory syndrome corona virus disease from
Saudi Arabia: a descriptive study. Lancet Infect Dis. doi:10.1016 (2013).

19. Root HF. The association of diabetes and tuberculosis. $N$ Engl J Med. 1934; 1: 210.

20. Jeon CY, Murray MB. Diabetes mellitus increases the risk of active tuberculosis: a systematic review of 13 observational studies. PLoS Med. 2008; 5: e152. * (provides an overview of studies linking increased risk of TB among people with $\mathrm{DM})$.

21. Stevenson CR, Crichley JA, Forouhi NG, et al. Diabetes and the risk of tuberculosis: a neglected threat to public health. Chronic IIIn 2007; 3: 228-45.

22. Stevenson CR, Forouhi NG, Roglic G, et al. Diabetes and tuberculosis: the impact of the diabetes epidemic on tuberculosis incidence. BMC Public Health 2007; 7: 234. doi:10.1186/1471-2458-7-234

23. Baker MA, Harries AD, Jeon $\mathrm{CY}$, et al. The impact of diabetes on tuberculosis treatment outcomes: A systematic review. BMC Med. 2011; 9: 81.** (reviews studies that link TB treatment outcomes with presence of DM)

24. Faurholt-Jepsen D, Range N, PrayGod G, et al. Diabetes is a strong predictor of mortality during TB treatment. A prospective cohort study amongst TB patients from Mwanza,Tanzania. Trop Med Int Health 2013; 18: 822-9.

25. Jimenez-Corona M, Cruz-Hervert L, Garcia L, et al. Association of diabetes and tuberculosis: impact of outcomes and transmissibility to household contacts. Int J Tuberc Lung Dis. 2012; 16: S335-S6.

26. Kao LS, Knight MT, Lally KP, Mercer DW. The impact of diabetes in patients with necrotizing soft tissue infections. Surg Infect (Larchmt) 2005; 6: 427-38.

27. Sendi $P$, Johansson L, Norrby-Teglund A. Invasive group B Streptococcal disease in non-pregnant adults: a review with emphasis on skin and soft-tissue infections. Infection 2008; 36: 100-111.

28. Homer-Vanniasinkam S. Surgical site and vascular infections: treatment and prophylaxis. Int J Infect Dis. 2007; 11: S17S22.

29. DiNubile MJ, Lipsky BA. Complicated infections of skin and skin structures: when the infection is more than skin deep. J Antimicrob Chemother 2004; 53(Suppl 2): ii37-ii50.

30. Lipsky BA, Berendt AR, Deery HG, et al. Diagnosis and treatment of diabetic foot infections. Clin Infect Dis. 2004; 39: 885-910.

31. Rajagopalan S. Serious infections in elderly patients with diabetes mellitus. Clin Infect Dis. 2005; 40: 990-6.

32. Ramakant $\mathrm{P}$, Verma K, Misra R, et al. Changing microbiological profile of pathogenic bacteria in diabetic foot infections: time for a rethink on which empirical therapy to choose? Diabetologia 2011; 54: 58-64.

33. Dang CN, Prasad YD, BoultonAJ, Jude EB. Methicillin resistant Staphylococcus aureus in the diabetic foot clinic: a worsening problem. Diabet Med. 2003; 20: 159-61.

34. Stanaway S, Johnson D, Moulik P, Gill G. Methicillin resistant Staphylococcus aureus (MRSA) isolation from diabetic foot ulcers correlates with nasal MRSA carriage. Diabetes Res ClinPract 2007; 75: 47-50. 
35. Vardakas KZ, Horianopoulou M, Falagas ME. Factors associated with treatment failure in patients with diabetic foot infections: an analysis of data from randomized controlled trials. Diabetes Res Clin Pract 2008; 80: 344-51.

36. Abbas ZG, Archibald LK. Tropical diabetic hand syndrome. Epidemiology, pathogenesis and management. Am J Clin Dermatol 2005; 6: 21-28.

37. Lipsky BA. Medical treatment of diabetic foot infections. Clin Infect Dis. 2004; 39: S104-S114

38. de Lastours V, Foxman B. Urinary tract infection in diabetes: epidemiologic considerations. Curr Infect Dis Rep. 2014; 16(1): 389. doi:10.1007/s11908-013-0389-2

39. Geerlings S, Fonseca V,Castro-Diaz D, List JF, Parikh SJ. Genital and Urinary tract infections in diabetes: Impact of pharmacologically-induced glucosuria. Diabetes Res Clin Pract 2014; PII: S0168-8227(14)00013-8, doi:10.1016/ j.diabres.2013.12.052.

40. Johnsson KM, Ptaszynska A, Schmitz B, Sugg J, Parikh SJ, List JF. Urinary tract infections in patients with diabetes treated with dapagliflozin. J Diabetes Complications 2013; 27: 473-8.

41. Jackson SL, Boyko EJ, Scholes D, Abraham L, Gupta K, Finn SD. Predictors of urinary tract infection after menopause: a prospective study. Am J Med 2004; 117: 903-11.

42. Horcajada JP, Moreno I, Velasco M, et al. Communityacquired febrile urinary tract infection in diabetics could deserve a different management: a case-control study. $J$ Intern Med. 2003; 254: 280-6.

43. Bonadio M, Costarelli S, Morelli G, Tartaglia T. The influence of diabetes mellitus on the spectrum of uropathogens and the antimicrobial resistance in elderly adult patients with urinary tract infection. BMC Infectious Diseases. 2006; 6 : 54.

44. Vogel JP, Lee ACC, Souza JP. Maternal morbidity and preterm birth in 22 low- and middle-income countries: a secondary analysis of the WHO Global Survey dataset. BMC Pregnancy and Childbirth 2014; 14: 56. doi:10.1186/1471-2393-14-56.

45. Richards D. Oral Diseases affect some 3.9 Billion people. Evidence-Based Dentistry 2013; 14: 35. doi:10.1038/sj.ebd. 6400925

46. Mealey BL, Ocampo GL. Diabetes mellitus and periodontal disease. Periodontol 2000 2007; 44: 127-53.

47. Borgnakke WS, Ylöstal PV, Taylor GW, Genco RJ. Effect of periodontal diseaseon diabetes: systematic review of epidemiologic observational evidence. J Clin Periodontol 2013; 40(Suppl. 14), S135-S152.

48. Preshaw PM, Alba AL, Herrera D, et al. Periodontitis and diabetes: a two-way relationship. Diabetologia 2012; 55: 21-31.

49. Engebretson S, Kocher T. Evidence that periodontal treatment improves diabetes outcomes: a systematic review and metaanalysis. J Clin Periodontol 2013; 40: S153-S163.* (presents evidence that treatment of periodontitis is associated with significant improvement in metabolic control almost amounting to the same level as may be achieved by adding an additional anti-diabetic drug).

50. Simon L, Tornóczky J, Tóth M, Jámbor M, Sudár Z. The significance of Campylobacter pylori infection in gastroenterologic and diabetic practice. Orv Hetil 1989; 130: 1325-9.

51. Wang F, Liu J, Lv Z. Association of Helicobacter pylori infection with diabetes mellitus and diabetic nephropathy: a meta-analysis of 39 studies involving more than 20,000 participants. Scand J Infect Dis. 2013; 45: 930-8.

52. Hsieh MC, Wang SS, Hsieh YT, Kuo FC, Soon MS, Wu DC. Helicobacter pylori infection associated with high $\mathrm{HbA1c}$ and type 2 diabetes. Eur J Clin Invest. 2013; 43, 949-56.

53. Jeon $C Y$, Haan $M N$, Cheng $C$, et al. Helicobacter pylori infection is associated with an increased rate of diabetes. Diabetes Care 2012; 35: 520-5. 